\title{
Charge Transport in Rubrene-Based Crystals
}

\author{
1,*Hudson Rodrigues Armando (PG), ${ }^{2}$ Denis da Mata Oliveira (PG), and ${ }^{3}$ Luiz Antônio \\ Ribeiro Júnior (PQ) \\ ${ }^{1}$ University of Brasília, PPG-CIMA, Campus Planaltina, 73345-010, Brasília, DF, Brazil, ${ }^{2}$ Institute of \\ Physics, University of Brasília, Brasília, 70910-970, DF, Brazil and ${ }^{3}$ International Center for Condensed \\ Matter Physics, University of Brasília, P.O. Box 04531, 70.919-970, Brasília, DF, Brazil \\ Keywords: Organic Semiconductors, Rubrene, Monte Carlo, Charge Transport.
}

\section{Introduction}

The need of understanding the underlying properties of the charge transport in organic semiconductors has driven the development of microscopic numerical models [1]. Especially simulations based on kinetic Monte Carlo (KMC) techniques provide a powerful and versatile tool to gain further insight into these mechanisms because they allow to represent the complex morphology of bulk-heterojunction blends and to track the dynamic behaviour of single particles [1]. Here, the transport of holes in rubrene-based crystals is theoretically studied in the framework of a model based on the KMC algorithm. A good model-to-experiment agreement with realistic material parameters as input values validates the model.

\section{Methodology}

Within the hopping description, the charge transport can be characterized by a diffusion process, in which the charge carrier hops between adjacent molecules according to the charge transfer rates that apply in the absence of an external electric field [3]. In order to account the charge transfer rates, we have implemented in our KMC code the widely used charge transfer rate from the classical Marcus theory [3]. An overall description about our KMC method can be found in the reference [3].

\section{Results}

With KMC simulations, we have calculated the mobility dependence on temperature and electric field for different rubrene-based crystals. The obtained mobilities present values that are much closer to the experimental values, about $1 \mathrm{~cm}^{2} /(\mathrm{Vs})$, still maintaining the same qualitative functional dependence of the mobility on the electric field.

\section{Conclusions}

A realistic $\mathrm{KMC}$ model for charge transport in organic materials is obtained here. Our KMC method satisfactorily model the transport of several charge carriers via hopping between localized states. The implementation of long-range electrostatic forces by evaluation of the Ewald sum allows to accurately represent the charge 
interactions in low-dielectric organic materials and makes the model unique in its features. The good agreement between theory and experiment, when it comes to the mobility values, indicates that our KMC model is able to reproduce accurately the charge transport mechanism in real devices.

\section{Acknowledgements}

The authors gratefully acknowledge the financial support from the Brazilian Research Councils CAPES and FAPDF. L.A.R.J. gratefully acknowledges the financial support from the Brazilian Research Council FAPDF grant 0193.000942/2015 and 193.001.511/2017. L.A.R.J. also wishes to thank the Brazilian Ministry of Planning, Budget and Management (Grant DIPLA 005/2016).

\section{References}

[1] J. L. Brédas et. al. Acc. Chem. Res. 42, 1691-1699, 2009.

[2] V. Coropceanu et. al. Chem. Rev. 107, 926-952, 2007.

[3] R. Volpi Modeling Charge Transport for Organic Solar Cells within Marcus Theory. PhD Thesis, Linköping University, Linköping, Sweden 2017. 